\title{
ASTEROSEISMIC-BASED ESTIMATION OF THE SURFACE GRAVITY FOR THE LAMOST GIANT STARS
}

\author{
Chao Liu ${ }^{1}$, Min Fang ${ }^{2,3}$, Yue Wu ${ }^{1}$, Li-Cai Deng ${ }^{1}$, Liang Wang ${ }^{1}$, Wei Wang ${ }^{1}$, Jian-Ning Fu ${ }^{4}$, Yong-Hui Hou ${ }^{5}$, \\ GuANG-Wei Li ${ }^{1}$, AND YONG ZHANG ${ }^{5}$ \\ ${ }^{1}$ Key Laboratory of Optical Astronomy, National Astronomical Observatories, Chinese Academy of Sciences, 20 A Datun Road, Beijing 100012, China; \\ liuchao@nao.cas.cn \\ ${ }^{2}$ Departamento de Física Teórica, Facultad de Ciencias, Universidad Autonóma de Madrid, E-28049 Cantoblanco, Madrid, Spain \\ Purple Mountain Observatory and Key Laboratory for Radio Astronomy, 2 West Beijing Road, 210008, Nanjing, China \\ ${ }^{4}$ Department of Astronomy, Beijing Normal University, 19 Avenue Xinjiekouwai, Beijing 100875, China \\ ${ }^{5}$ Nanjing Institute of Astronomical Optics \& Technology, National Astronomical Observatories, Chinese Academy of Sciences, Nanjing 210042, China \\ Received 2014 November 2; accepted 2015 May 8; published 2015 June 24
}

\begin{abstract}
Asteroseismology is one of the most accurate approaches to estimate the surface gravity of a star. However, most of the data from the current spectroscopic surveys do not have asteroseismic measurements, which is very expensive and time consuming. In order to improve the spectroscopic surface gravity estimates for a large amount of survey data with the help of the small subset of the data with seismic measurements, we set up a support vector regression (SVR) model for the estimation of the surface gravity supervised by 1374 Large Sky Area Multi-object Fiber Spectroscopic Telescope (LAMOST) giant stars with Kepler seismic surface gravity. The new approach can reduce the uncertainty of the estimates down to about 0.1 dex, which is better than the LAMOST pipeline by at least a factor of 2 , for the spectra with signal-to-noise ratio higher than 20. Compared with the $\log g$ estimated from the LAMOST pipeline, the revised $\log g$ values provide a significantly improved match to the expected distribution of red clump and red giant branch stars from stellar isochrones. Moreover, even the red bump stars, which extend to only about 0.1 dex in $\log g$, can be discriminated from the new estimated surface gravity. The method is then applied to about 350,000 LAMOST metalrich giant stars to provide improved surface gravity estimates. In general, the uncertainty of the distance estimate based on the SVR surface gravity can be reduced to about $12 \%$ for the LAMOST data.
\end{abstract}

Key words: asteroseismology - methods: data analysis - methods: statistical - stars: fundamental parameters

\section{INTRODUCTION}

The surface gravity of a star is an important stellar astrophysical parameter in the sense that it is able to measure the radius of a star given the stellar mass. Together with the effective temperature and metallicity, a star can be pinned down in the Hertzsprung-Russell diagram with the surface gravity. This will be very helpful to learn the evolution status of the star, as well as its distance. Therefore, an accurate estimation of the surface gravity is critical in the study of either stars or stellar systems.

Although multiband photometry may help to discriminate giant from dwarf stars according to some surface-gravitysensitive features (Lenz et al. 1998; Majewski et al. 2000; Yanny et al. 2000), spectroscopic data can reveal more detailed features to quantify the surface gravity. First, the prominent $\mathrm{Mg} b+\mathrm{MgH}$ feature observed in low-resolution spectra is used not only to identify giant stars (Liu et al. 2014; Xue et al. 2014), but also to measure their surface gravity (Morrison et al. 2003; Lee et al. 2008). Second, the other features, e.g., Balmer lines (Wilhelm et al. 1999), Ca II, K, and H lines (Lee et al. 2008), etc., can also be useful to the determination of the surface gravity. Moreover, some algorithms determine the surface gravity together with the effective temperature and metallicity simultaneously, by comparing the full spectra with the spectral library (e.g., Lee et al. 2008; Wu et al. 2011b). In addition, the supervised machine learning approaches, e.g., artificial neural networks, support vector machine (SVM), etc., have also been used to derive the surface gravity based on the training spectra with known surface gravity values as the targets (Re Fiorentin et al. 2006; Liu et al. 2012). The typical accuracy of the surface gravity estimates for low-resolution spectra, e.g., the Sloan Digital Sky Survey (SDSS; Ahn et al. 2014) or the Large Sky
Area Multi-object Fiber Spectroscopic Telescope (LAMOST; Cui et al. 2012), is about 0.2-0.4 dex (Wilhelm et al. 1999; Re Fiorentin et al. 2006; Lee et al. 2008; Wu et al. 2014).

Asteroseismology is a powerful tool to derive the fundamental parameters, e.g., stellar mass, radius, and $\log g$, for a star (see Brown \& Gilliland 1994; Chaplin \& Miglio 2013). Thanks to the Kepler (Borucki et al. 2010) mission, the solarlike oscillations for tens of thousands of stars are able to be measured. The surface gravity can be estimated from the oscillations with accuracy of $0.02-0.05 \mathrm{dex}$ (Morel \& Miglio 2012; Creevey et al. 2013). This performance is much better than the non-seismic methods from even the highresolution spectra. Indeed, Epstein (2014b) has shown that the Kepler-measured seismic $\log g$ is more accurate than those from the high-resolution infrared APOGEE (Majewski et al. 2010) spectra by a factor of a few.

However, compared to the huge amount of spectra from many large spectroscopic survey projects, the number of stars with asteroseismic measurement is still very limited. Therefore, it is crucial to examine how the surface gravity of all the spectroscopic survey data can be improved with the existing seismic data, which only occupies a small fraction of the full samples. In this paper, we give more accurate $\log g$ estimates for the LAMOST data with the help of a small subset of the spectra with Kepler seismic $\log g$.

LAMOST, also known as the Guoshoujing Telescope, is a new type of $5^{\circ}$ wide field telescope with a large aperture of $4 \mathrm{~m}$. It assembles 4000 fibers on its large focal plane and can simultaneously observe a similar number of low-resolution $(R \sim 1800)$ spectra covering the wavelength from 380 to $900 \mathrm{~nm}$ (Cui et al. 2012; Zhao et al. 2012). As its main scientific goal, it will observe a few millions of stellar spectra with limiting magnitude down to $r \sim 18 \mathrm{mag}$ for diverse 
studies of the Milky Way (Deng et al. 2012). It has also sampled the Kepler field with the "LAMOST-Kepler project" (De Cat et al. 2014), in which a few thousand spectra with Kepler seismic data (Huber et al. 2014) are included. This small subset provides perfect calibrators to improve the estimation of $\log g$ for the LAMOST spectra.

In this work, we develop a support vector regression (hereafter SVR) model for the determination of the surface gravity for the LAMOST giant stars supervised by the data with Kepler seismic $\log g$. The paper is organized as follows. In Section 2, we give a detailed introduction to SVR. Then, we specifically set up an SVR model to estimate $\log g$ for LAMOST data in Section 3. The performance of the determination is also assessed in this session. Some discussions, including the systematics from the seismic $\log g$, the influences in various evolution phases of the stars, the metallicity effect, the comparison with other similar works, and the benefits and limits of this technique, are raised in Section 4. Finally, a brief conclusion is drawn in the final section.

\section{SUPPORT VECTOR REGRESSION}

SVM is a well-known supervised machine learning algorithm mostly in the application of classification and nonlinear regression (Cortes \& Vapnik 1995; Burges 1998; Deng et al. 2012). SVR (Drucker et al. 1996), as an extension of the SVM, is a regression method by transforming the data, via a kernel function, from the nonlinear physical space into a highdimensional inner-product space, in which a linear model to the data can be fitted.

We refer the reader to Smola \& Schölkopf (2004) for a complete description of SVR. We only give an outline of the approach. Assume that $\left\{\left(\boldsymbol{x}_{1}, y_{1}\right),\left(\boldsymbol{x}_{2}, y_{2}\right), \ldots,\left(\boldsymbol{x}_{\ell}, y_{\ell}\right)\right\}$ are the training data, where $\boldsymbol{x}_{i}$ is the input vector in $d$ dimensions and $y_{i}$ is the corresponding known output. Because the relationship between $x$ and $y$ is highly nonlinear, the SVR is to find a map, $\Phi$, mapping the real input space to a higher-dimensional feature space, in which the nonlinear regression problem can be converted into a linear problem. For this purpose, the SVR constructs a form such as

$$
f(\boldsymbol{x})=\sum_{i}^{\ell}\left\langle\left(\alpha_{i}-\alpha_{i}^{*}\right) \Phi\left(\boldsymbol{x}_{i}\right), \Phi(\boldsymbol{x})\right\rangle+b,
$$

where $\langle\cdot, \cdot\rangle$ is the inner product and $\alpha_{i}, \alpha_{i}^{*}$, and $b$ are parameters to be solved. Practically, $\Phi$ is not explicitly given, but uses a kernel function, $K\left(\boldsymbol{x}, \boldsymbol{x}^{\prime}\right) \equiv\left\langle\Phi(x), \Phi\left(x^{\prime}\right)\right\rangle$, instead. Among lots of choices of the kernels, the radial-based kernel, which has the form of $\exp \left(-\gamma\left\|\boldsymbol{x}-\boldsymbol{x}^{\prime}\right\|\right)$, is often used. Mathematically, it can be proved that $f$ is linear in the innerproduct space. The solution of Equation (1) can be derived from a convex optimization problem:

$$
\begin{array}{ll}
\operatorname{minimize} & \frac{1}{2}\|\boldsymbol{w}\|^{2}+C \sum_{i=1}^{\ell}\left(\xi_{i}+\xi_{i}^{*}\right) \\
\text { subject to } & \left\{\begin{array}{l}
y_{i}-\left\langle\boldsymbol{w}, \Phi\left(\boldsymbol{x}_{i}\right)\right\rangle-b \leqslant \epsilon+\xi_{i} \\
\left\langle\boldsymbol{w}, \Phi\left(\boldsymbol{x}_{i}\right)\right\rangle+b-y_{i} \leqslant \epsilon+\xi_{i}^{*} \\
\xi_{i}, \xi_{i}^{*} \geqslant 0
\end{array}\right.
\end{array}
$$

where $w=\sum_{i=1}^{\ell}\left(\alpha_{i}-\alpha_{i}^{*}\right) \Phi\left(\boldsymbol{x}_{i}\right), C>0$ is the cost parameter to determine the trade-off between the flatness of $f$ and the

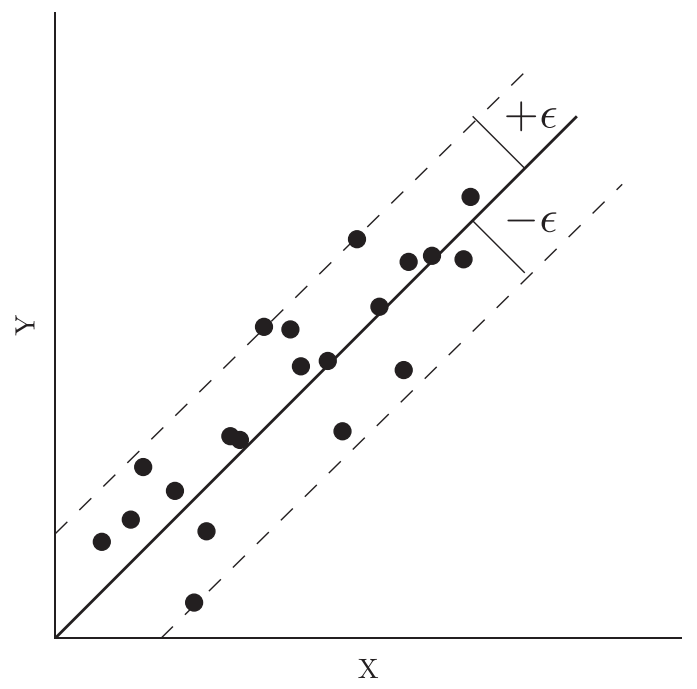

Figure 1. Demonstration of how the SVR model works in the linear case. The black dots are the mock data with arbitrary scales in $x$ - and $y$-axes. The solid line stands for the SVR model, and the dashed lines show the tolerance of $\epsilon$ on each side of the linear model.

amount up to which deviations larger than $\epsilon$ are tolerated, and $\xi_{i}$ and $\xi_{i}^{*}$ are slack variables. Figure 1 demonstrates how the SVR works in the linear case.

Chang \& Lin (2011) provide a multi-programming language package, LIBSVM, ${ }^{6}$ to solve the SVR model based on Equations (1) and (2), as well as other SVM models. The process of finding the optimized solution of the SVR model contains two steps. The first step is to determine the parameters $C, \epsilon$, and $\gamma$ from $n$-fold cross-validations. In this step, the software randomly divides the training data set into $n$ groups with equal size and predicts the dependent values for each group using the SVR model trained by the remaining $n-1$ groups of data. The best choices of $C, \epsilon$, and $\gamma$ are those that give the best cross-validation accuracy of the regression. The second step is to find the optimal solution of $\alpha_{i}, \alpha_{i}^{*}, \xi_{i}, \xi_{i}^{*}$, and $b$ in Equation (2) for the whole training data set using the best choices of $C, \epsilon$, and $\gamma$. Then, the derived SVR model, Equation (1), can be used to predict the corresponding dependent variable for given input data. A typical application of the SVR is also found in Liu et al. (2012).

\section{ESTIMATE LOG $G$ FOR LAMOST SPECTRA}

\subsection{The Training and Test Data Set}

In order to establish an SVR model for the $\log g$ determination based on the seismic estimates, we first select a proper training data set. Huber et al. (2014) released $\sim 200,000$ stars with stellar parameters in the Kepler field, among which 15,686 stars have surface gravity estimated from asteroseismology (hereafter, we call it the Huber catalog). We use the parameters listed in Table 4 of Huber et al. (2014), which is directly compiled from literature and not fitted to the model isochrones. We cross-identify these stars with the LAMOST DR2 data and obtain 3335 common stars with the signal-to-noise ratio $(\mathrm{S} / \mathrm{N})$ at $g$ band higher than 10 in the

\footnotetext{
6 http://www.csie.ntu.edu.tw/ cjlin/libsvm/
} 


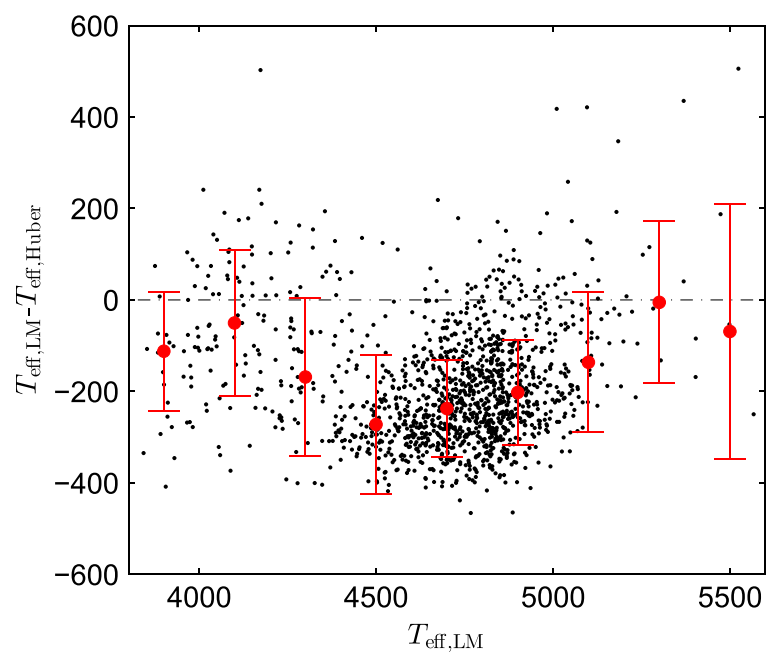

Figure 2. Difference between the LAMOST $T_{\text {eff }}$ and the $T_{\text {eff }}$ in the Huber catalog. The $x$-axis is the LAMOST $T_{\text {eff }}$, and they-axis is the difference of the $T_{\text {eff }}$ between LAMOST and the Huber catalog. The black dots stand for the individual stars in the training data set, and the red filled circles with error bars indicate the medians and dispersions of the differences at various $T_{\text {eff }}$ bins.

spectra. $^{7}$ The stellar parameters of these LAMOST spectra are estimated in the pipeline using the software ULySS, with the empirical stellar library ELODIE as a reference (Wu et al. 2011a, 2011b, 2014).

The scaling relation of the seismic $\log g$, as shown in Equation (3), depends on $T_{\text {eff }}$ :

$$
\frac{g}{g_{\odot}} \cong\left(\frac{\nu_{\max }}{\nu_{\max , \odot}}\right)\left(\frac{T_{\text {eff }}}{T_{\text {eff } \odot}}\right)^{0.5},
$$

where $\nu_{\max }$ is the frequency of maximum power and the quantities with $\cdot \odot$ stand for the solar parameters (Chaplin \& Miglio 2013). The effective temperature used to derive the seismic $\log g$ in the Huber catalog may not be consistent with the LAMOST $T_{\text {eff }}$. Indeed, Figure 2 shows that the LAMOST $T_{\text {eff }}$ is smaller than those in the Huber catalog by about $200 \mathrm{~K}$. Therefore, the initial seismic $\log g$ in the Huber catalog needs to be recalibrated with LAMOST $T_{\text {eff }}$. Without providing the frequencies of the seismic oscillation in the Huber catalog, we can recalibrate the seismic $\log g$ by applying the following rescaling:

$$
g_{\text {ast,corr }}=g_{\text {ast, Huber }}\left(\frac{T_{\text {eff,LM }}}{T_{\text {eff,Huber }}}\right)^{0.5},
$$

where $T_{\text {eff,LM }}$ is the LAMOST-derived effective temperature and $T_{\text {eff,Huber }}$ and $\log g_{\text {ast,Huber }}$ are the effective temperature and the seismic surface gravity provided by the Huber catalog, respectively. In order to keep self-consistence of $T_{\text {eff }}$ in the Huber catalog, we only use the objects with Pinsonneault et al. (2012) $T_{\text {eff }}$ or whose $T_{\text {eff }}$ is calibrated with Pinsonneault et al. (2012) in the Huber catalog. Consequently, a very small fraction of stars, which are from the references 6, 13, and 14 and hence may not explicitly calibrate to Pinsonneault et al.

\footnotetext{
7 We use the $\mathrm{S} / \mathrm{N}$ at $g$ band because most of the spectral lines sensitive to $\log g$ are located in this range of wavelength (Liu et al. 2014).
}

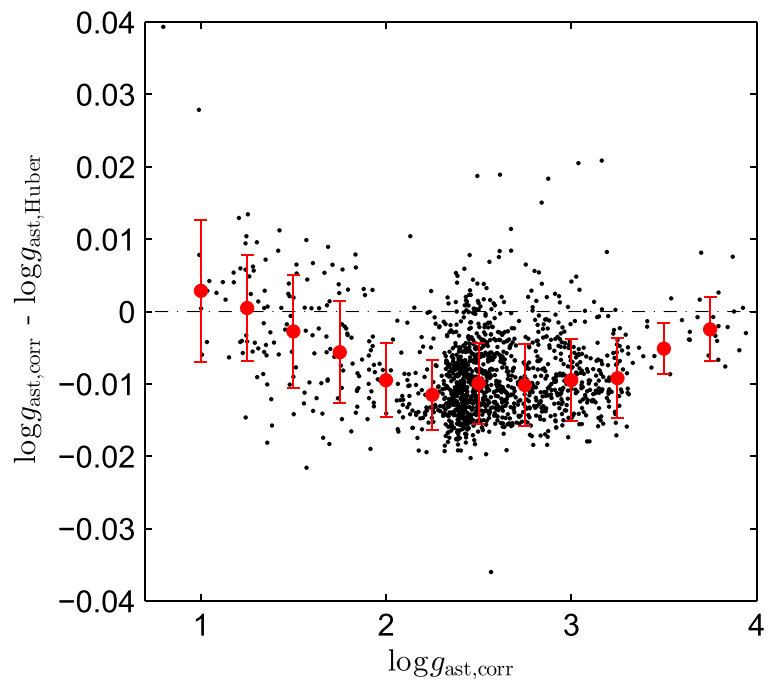

Figure 3. Difference between the initial $\log g$ from the Huber catalog and the recalibrated one using LAMOST $T_{\text {eff,LM }}$. The $x$-axis is the recalibrated $\log g$, while the $y$-axis is the difference. The black dots stand for the stars in the training data set, and the red filled circles with error bars are the medians and dispersions of the differences at various $\log g_{\text {ast,corr }}$ bins.

(2012), in the Huber catalog are excluded from our training and test data set.

Figure 3 shows the difference between the initial and the recalibrated $\log g$. The recalibrated $\log g$, denoted as $\log g_{\text {ast,corr }}$, is slightly smaller than the initial $\log g$, denoted as $\log g_{\text {ast,Huber }}$, by only about $0.01 \mathrm{dex}$, which is essentially within the uncertainty of the seismic $\log g$. This trend does naturally follow the difference in $T_{\text {eff }}$ between the two catalogs. Although this tiny difference would not significantly affect our result, we still adopt the recalibrated $\log g$ in this work to keep well self-consistency between the LAMOST $T_{\text {eff }}$ and the seismic $\log g$. In the rest of the paper, unless explicitly indicated, we refer to the recalibrated seismic $\log g$ by default, when we use $\log g_{\text {ast }}$.

Then, we select the metal-rich giant stars with $\quad 3500<T_{\text {effLM }}<6000 \mathrm{~K}, \quad \log g_{\mathrm{LM}}<4.0 \mathrm{dex}, \quad$ and $[\mathrm{Fe} / \mathrm{H}]_{\mathrm{LM}}>-0.6$ dex, where the subscript "LM" denotes that the parameters are from the LAMOST pipeline. The selection in metallicity is because the current scaling relation in asteroseismology may not be suitable for the metal-poor stars, e.g., the thick-disk or the halo stars (Epstein et al. 2014a). The selection in $\log g_{\mathrm{LM}}$ is motivated by the small amount of seismic detections for dwarf stars in the Kepler database. Finally, we select 2726 stars with both seismic $\log g$ and LAMOST spectra with $U L y S S$-derived $T_{\text {eff,LM }},[\mathrm{Fe} / \mathrm{H}]_{\mathrm{LM}}$, and $\log g_{\text {LM }}$.

First, the data are arbitrarily separated into two groups with essentially equal members. One, known as the training data set (1374 stars), is used to train the SVR to find the best-fit model; the other, including the remaining 1352 stars, is used as the test data set to assess the performance.

Second, we define the proper quantities as inputs of the SVR model. We use the equivalent widths of 23 Lick lines (Worthey et al. 1994; Worthey \& Ottaviani 1997), including $\mathrm{H}_{\delta}, \mathrm{H}_{\gamma}, \mathrm{H}_{3}$, $\mathrm{H}_{\alpha}, \mathrm{Mg}_{1}, \mathrm{Mg}_{2}, \mathrm{Mg}_{b}$, Fe (4383, 4531, 4668, 5015, 5270, 5335, 5406, 5709, $5782 \AA), \mathrm{CN}, \mathrm{Ca}(4227,4455 \AA), G$ band, Na D, and $\operatorname{TiO}(5950,6187-6269 \AA)$, measured from the LAMOST spectra, rather than the full spectra, as the input to the SVR 
model. These line indices are more robust to the noise than the full spectrum.

In general, if the line indices vary with $\log g$ in the same way as with $T_{\text {eff }}$, then it is not easy to distinguish whether the change of the line indices is caused by the change of $\log g$ or $T_{\text {eff }}$. This is the so-called degeneracy between $\log g$ and $T_{\text {eff }}$. For instance, $\mathrm{H}_{\alpha}, \mathrm{H}_{3}$, and $\mathrm{TiO}$ show similar trends of variation when either $\log g$ or $T_{\text {eff }}$ changes (see Figures 4 and 5). However, not all of the 23 Lick line indices are affected by the degeneracy. The lines such as $\mathrm{Mg}_{1}, \mathrm{Mg}_{2}, \mathrm{Mg}_{b}, \mathrm{Fe} 5335, \mathrm{Fe} 5782$, etc., vary with $\log g$ in a different way with $T_{\text {eff }}$ (also see Figures 4 and 5). Therefore, these lines are more helpful to break the degeneracy and allow us to estimate $\log g$. Figure 6 also shows that the variations of $[\mathrm{Fe} / \mathrm{H}]$ in the line indices are quite different with $\log g$, meaning that there is relatively little degeneracy between $[\mathrm{Fe} / \mathrm{H}]$ and $\log g$.

Although the SVR model can only deal with a onedimensional dependent variable, it may not produce systematically biased $\log g$ estimates related to either $T_{\text {eff }}$ or $[\mathrm{Fe} / \mathrm{H}]$, because the training data set covers the same ranges of the metallicity and effective temperature as the test data set and the full LAMOST sample. More investigations will be discussed in Section 3.2.

It is also worthwhile to notice that although the asteroseismic $\log g_{\text {ast }}$ is used as the known dependent variable in the training data set, it does not mean that the seismic $\log g$ is the true value for a star. It is also affected by some systematics, as mentioned later in Section 4.1. However, compared to the relatively larger uncertainty of the spectroscopic $\log g$ estimates, the systematic bias in the seismic $\log g$ is quite small and can be negligible. Moreover, the motivation of this work is not to find the true $\log g$ for the spectra, but to find the better $\log g$ estimates than the other spectroscopic methods. Since the seismic $\log g$ is so far the best choice, the goal of this work is to calibrate the $\log g$ estimated from the low-resolution spectra to the best one, i.e., the seismic $\log g$.

Note that the LAMOST-pipeline-derived $\log g_{\mathrm{LM}}$ is only used in the initial data selection; it plays no role in the SVR model. In other words, the SVR-predicted $\log g_{\mathrm{SVR}}$ is independent of $\log g_{\mathrm{LM}}$.

\subsection{Performance}

In this section we use the test data set with 1352 stars, which do not appear in the training data set but have seismic $\log g$ and similar ranges of $T_{\text {eff }}$ and $[\mathrm{Fe} / \mathrm{H}]$ to the training data set, to investigate the performance. The recalibrated seismic $\log g$ ast is used as the standard value to be compared with in the whole performance assessment.

An intuitive assessment of the performance of the SVR model is to compare the SVR $\log g$ (denoted as $\log g_{\mathrm{SVR}}$ ) with other estimates in the $T_{\mathrm{eff}}-\log g$ diagram. Figure 7 shows three $T_{\text {eff }}-\log g$ diagrams for the test data set with $\mathrm{S} / \mathrm{N}(g)>20$; the $y$-axes are $\log g_{\mathrm{LM}}, \log g_{\text {ast }}$, and derived $\log g_{\mathrm{SVR}}$ from panels (a) to (c), respectively. The $x$-axes, $\log T_{\text {eff,LM }}$, in the three panels are all from the LAMOST pipeline. The red dashed lines show the isochrones (Marigo et al. 2008) with $[\mathrm{Fe} / \mathrm{H}]=-0.2$ dex at the age of 1,5 , and $10 \mathrm{Gyr}$, from top to bottom.

First, the largest difference between $\log g_{\mathrm{LM}}$ and the other two is that for the red giant branch (RGB) stars, which are located below the isochrones for $\log g_{\mathrm{LM}}$ (panel (a)). This can also be clearly seen in Figure 8, in which the difference of the $\log g_{\mathrm{SVR}}$ and $\log g_{\mathrm{LM}}$ is below the zero point by $0.5 \mathrm{dex}$ for stars with $\log g_{\mathrm{SVR}}<2$ dex. Although it cannot be used to justify whether or not the LAMOST result is correct, the discrepancy between $\log g_{\mathrm{LM}}$ and the isochrone does bring significant systematic bias when one determines the distance by comparing $\log g_{\mathrm{LM}}$ and $T_{\mathrm{eff}, \mathrm{LM}}$ with the synthetic isochrones. The seismic (panel (b)) and SVR (panel (c)) $\log g$, on the other hand, are consistent with the isochrones for the RGB stars.

Second, the most prominent feature in the $T_{\text {eff }}-\log g$ diagrams is the red clump/bump stars, ${ }^{8}$ which concentrate at around $\log g \sim 2.5$ dex and $\log T_{\text {eff }} \sim 3.68$. Compared with the seismic $\log g$, the LAMOST-derived red clump stars (panel (a)) show an obviously tilted shape, which is probably an artificial effect due to the incompleteness and sparseness of the stellar library used in the current LAMOST pipeline. As the most accurate measurement of $\log g$, the seismic $\log g$ shows the clear red bump located at $0.1-0.3$ dex below the more concentrated and horizontally elongated red clump stars $\left(\log g \sim 2.4 \mathrm{dex}, 3.64<\log T_{\text {eff }}<3.7\right)$ in panel (b). Encouragingly, the SVR $\log g$ shows similar features in panel (c): (1) the red clump is more concentrated than the LAMOST $\log g$ and also shows a slightly elongated shape at $3.65<\log T_{\text {eff }}<3.7$; (2) the red bump stars can be barely discriminated just 0.3 dex below the red clump at $\log T_{\text {eff }}$ $\sim 3.65$, although the dispersion is larger than the seismic $\log g$.

Figure 8 shows the difference between the SVR and LAMOST $\log g$. For the stars with $\log g_{\mathrm{SVR}}>2.5 \mathrm{dex}, \log g_{\mathrm{SVR}}$ is slightly smaller than $\log g_{\mathrm{LM}}$ by about $0.1 \mathrm{dex}$. For those with $\log g_{\text {SVR }}<2.5$ dex, $\log g_{\text {SVR }}$ is significantly smaller than $\log g$ LM by $\sim 0.5$ dex. This means that the LAMOST-provided $\log g$ may overestimate the $\log g$ for the upper part of the RGB stars.

Figure 9 demonstrates the difference of the SVR with the seismic $\log g$ as a function of the $\mathrm{S} / \mathrm{N}$ at $g$ band of the corresponding LAMOST spectra for the test data set. The two thin red lines in Figure 9 indicate the $1 \sigma$ dispersion of the difference, which are flat at about \pm 0.1 dex when $\mathrm{S} / \mathrm{N}(g)>20$. Although this uncertainty is still larger than the seismic measurement, it is significantly better than any other nonseismic estimation for the low-resolution spectra by a factor of 2-4. This explains why we can marginally distinguish the bump stars from the $T_{\text {eff }}-\log g$ diagram with SVR $\log g$ in panel (c) of Figure 7.

Figure 10 shows the performance of the SVR $\log g$ compared with the corresponding seismic $\log g$ using the test data set with $\mathrm{S} / \mathrm{N}(g)>20$. First, the comparisons between the residual $\log g$ (defined as $\log g_{\mathrm{SVR}}-\log g_{\text {ast }}$ ) do not show any strong correlation with the effective temperature, as shown in panels (a). Second, it shows very weak anticorrelation with $[\mathrm{Fe} / \mathrm{H}]$, i.e., the SVR $\log g$ is very slightly overestimated for stars with $[\mathrm{Fe} / \mathrm{H}] \sim-0.6$ dex and underestimated for those with $[\mathrm{Fe} / \mathrm{H}] \sim+0.2$ dex. This systematic bias is below 0.05 dex, within the dispersion of the residual of $\log g_{\mathrm{SVR}}$. Third, in panel (c), for stars with $\log g_{\text {ast }}>3 \mathrm{dex}$, the SVR $\log g$ is underestimated by $\sim 0.1$ dex. Then the residual of $\log g_{\text {SVR }}$ changes from negative to positive when $\log g$ ast changes from 3 to 2.25 dex. Considering that the dispersion of the residual in this range is slightly larger than $0.1 \mathrm{dex}$, this systematics is not

\footnotetext{
8 Red clump stars are stars in the evolution phase of helium core burning bump stars are the phase of first ascent RGB stars in which a slight drop in the luminosities occurs when the extremely thin hydrogen shell is crossing to the discontinuous region (Cassisi \& Salaris 1997).
} 

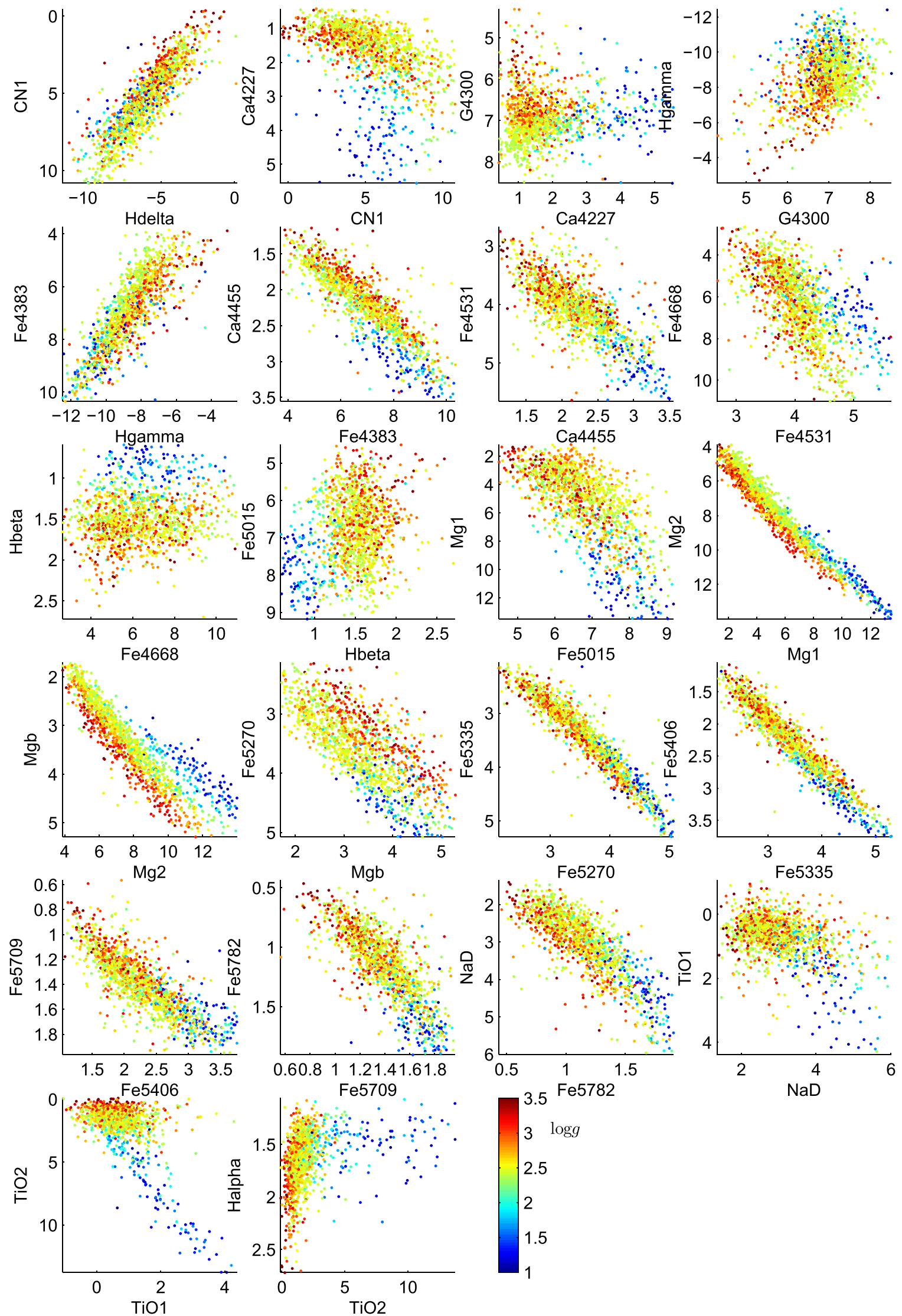

Figure 4. Correlations between $\log g_{\text {ast }}$ and the 23 Lick indices for the training data set. The $x$ - and $y$-axes are two different Lick indices, and the color codes give the corrected seismic surface gravity. 

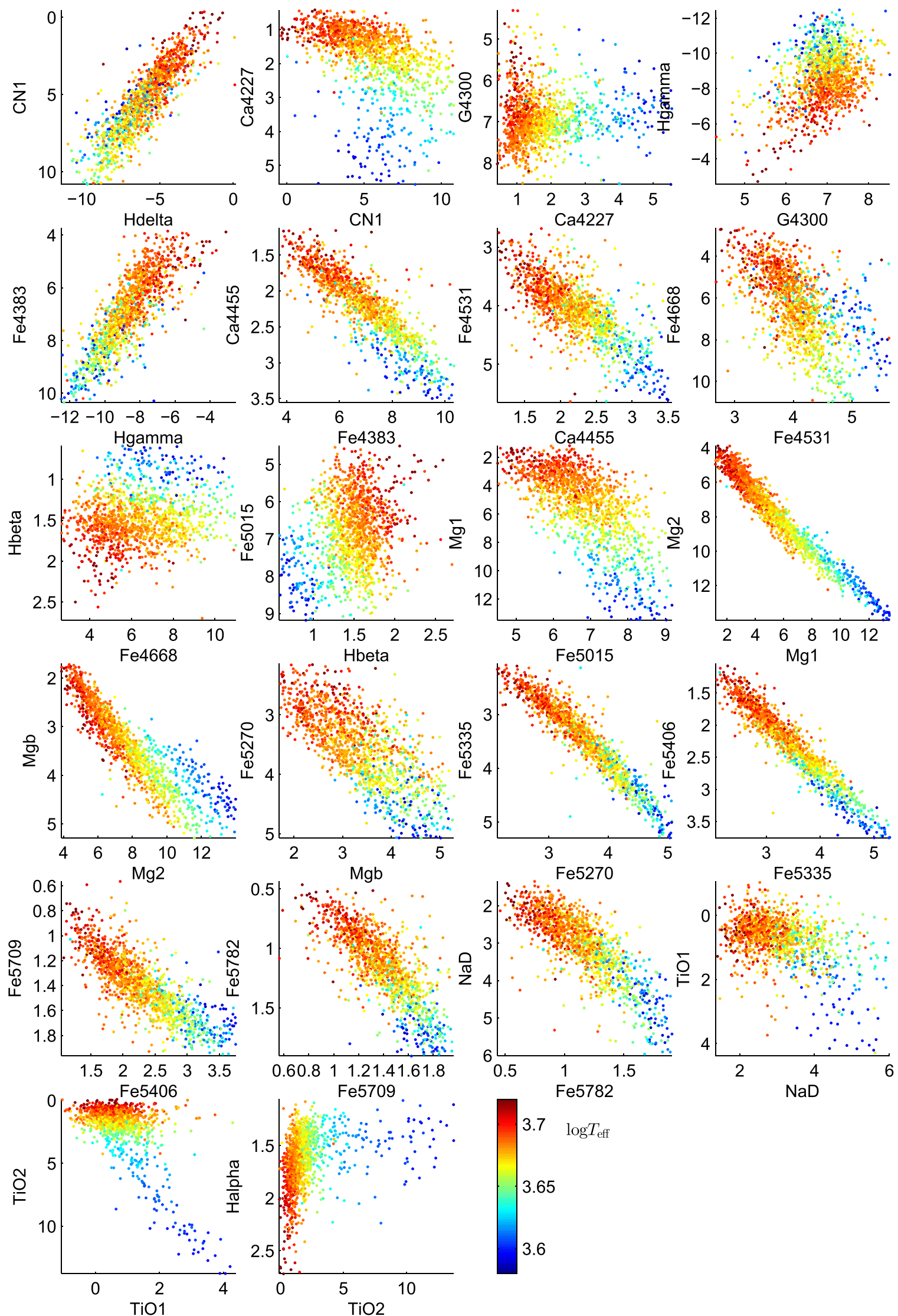

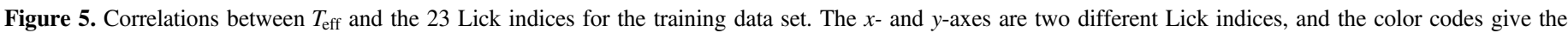
effective temperature from the LAMOST pipeline. 

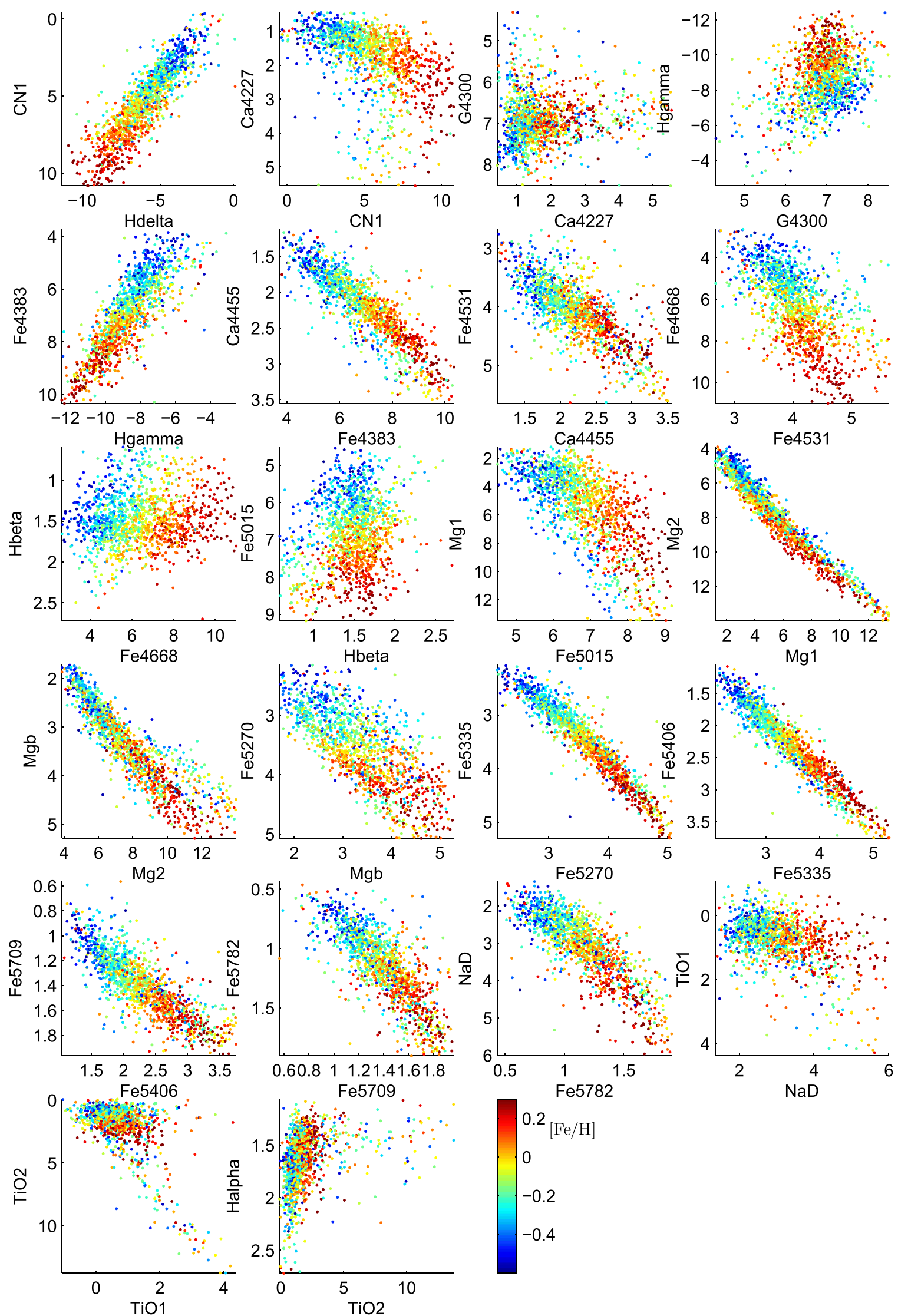

Figure 6. Correlations between $[\mathrm{Fe} / \mathrm{H}]$ and the 23 Lick indices for the training data set. The $x$ - and $y$-axes are two different Lick indices, and the color codes give the metallicity from the LAMOST pipeline. 

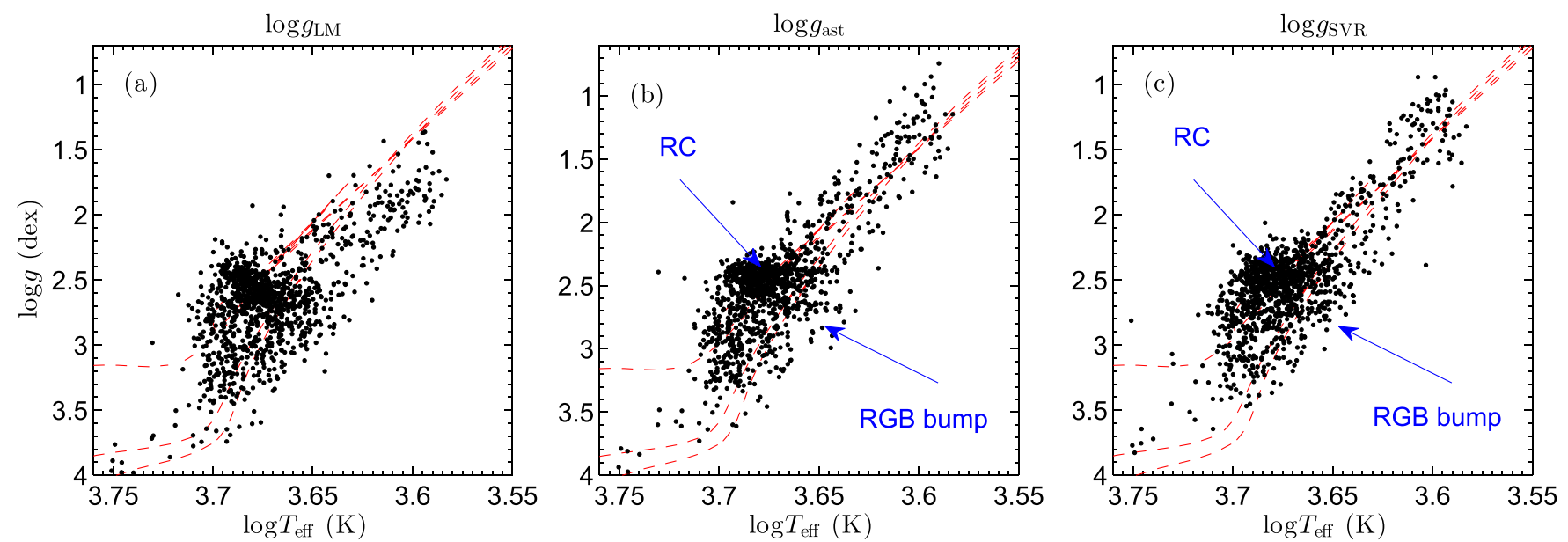

Figure 7. Distributions of the 1352-star test data set with $\mathrm{S} / \mathrm{N}(g)>20$ in $T_{\text {eff }}-\log g$ diagrams. The $\log g$ in panels (a), (b), and (c) are from the LAMOST pipeline, the

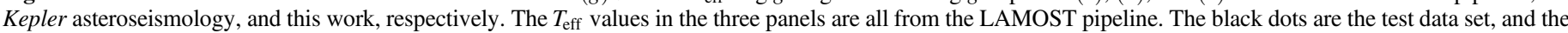
red dashed lines show the isochrones with $[\mathrm{Fe} / \mathrm{H}]=-0.2$ dex at the age of 1,5 , and $10 \mathrm{Gyr}$, from top to bottom. The red clump stars and the RGB bump stars in the second and third panels are indicated with text and arrows.

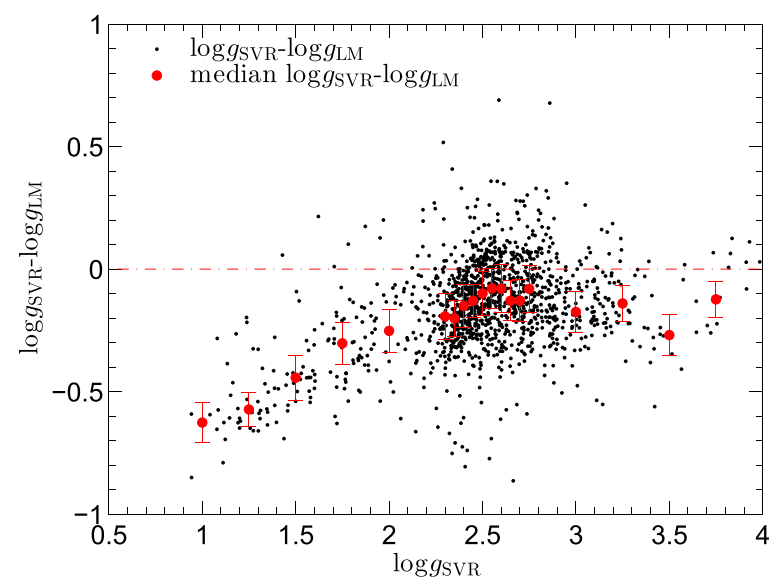

Figure 8. Comparison between the SVR and the LAMOST $\log g$ for the test data set (the black dots). The red filled circles with the error bars are the median values and $1 \sigma$ dispersions of the difference of $\log g$ at each $\log g$ ast bin.

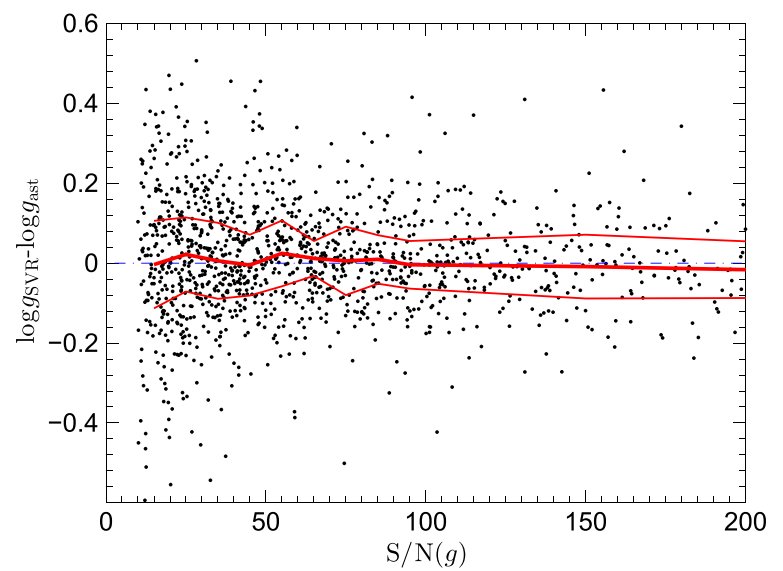

Figure 9. Variation of the residual $\log g$ of the test data set with the signal-tonoise ratio at $g$ band of the corresponding LAMOST spectra (the black dots). The thick red line is the median value, and the two thin red lines are the $1 \sigma$ of the dispersion. significant, although it is not negligible. For those $\log g_{\text {ast }}$ $<2.25 \mathrm{dex}$, a slight overestimation of less than $0.05 \mathrm{dex}$ in $\log g_{\text {SVR }}$ is found. This inverse S-shape in the residual $\log g_{\text {SVR }}$ versus $\log g$ ast is further discussed in Section 4.2. Finally, the distribution of the residual gives the overall uncertainty of the measurement in panel (d). The standard deviation for the residual is 0.16 dex. We also fit the distribution of the residual with a Gaussian to give an alternative estimation of the dispersion. The best-fit Gaussian (the red line) is centered at 0 , meaning that there is no overall systematic bias in the SVR estimation, and $\sigma$ is 0.11 dex.

\subsection{Apply to LAMOST Data}

We apply the SVR $\log g$ estimator to 356,932 selected LAMOST DR2 stars with $\log g_{\mathrm{LM}}<4$ dex, $[\mathrm{Fe} / \mathrm{H}]_{\mathrm{LM}}>-0.6 \mathrm{dex}$, and reliably measured equivalent widths for all 23 line indices. The final result is shown in panel (a) of Figure 11. As a comparison, panel (b) shows a similar plot to the LAMOST-derived $\log g$. Compared with the theoretical isochrones, the underestimation in the LAMOST $\log g$ of the RGB stars has been corrected in the results of this work, and the shape around the red clump stars now seems normal. However, a small fraction of stars with $\log T_{\text {eff }}>3.72$ are significantly underestimated in $\log g$ because of the poor quality of the corresponding spectra for these stars.

\section{DISCUSSIONS}

\subsection{The Systematic Bias in Seismic Parameter Determinations}

The SVR $\log g$ is automatically calibrated to the seismic $\log g$, since it uses the latter as the standard value in the training data set. This also means that all systematics that occur in seismic $\log g$ will also come into SVR $\log g$. Therefore, it is important that the users, particularly those who are not working in asteroseismology, of the SVR $\log g$ should be aware of the performance of the seismic $\log g$. Quite a lot of work has been done to investigate the performance of the seismic scaling relations. Here we just briefly review a few of them related to 


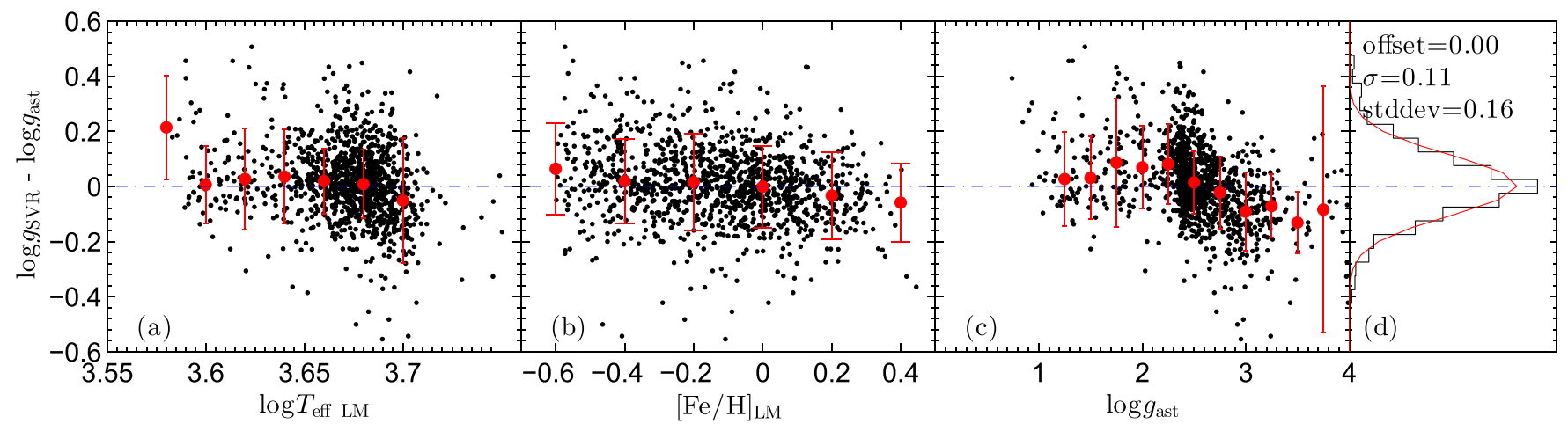

Figure 10. Residuals of the SVR $\log g, \log g_{\mathrm{SVR}}-\log g_{\text {ast }}$ for the test data set with $\mathrm{S} / \mathrm{N}(g)>20$ as functions of the LAMOST $\log T_{\text {eff }}$ and [Fe/H] are shown as the black dots in panels (a) and (b), respectively. Panel (c) shows the residual $\log g$ as a function of the seismic $\log g$ with the black dots. The red filled circles with error bars in the three panels indicate the medians and dispersions at various positions. Panel (d) shows the distribution of the residual log $g$ with the black line. The red line in this panel is the best-fit Gaussian, with parameters of offset $=0$ and $\sigma=0.11$ dex, to the residual. The standard deviation of the residual is 0.16 dex.

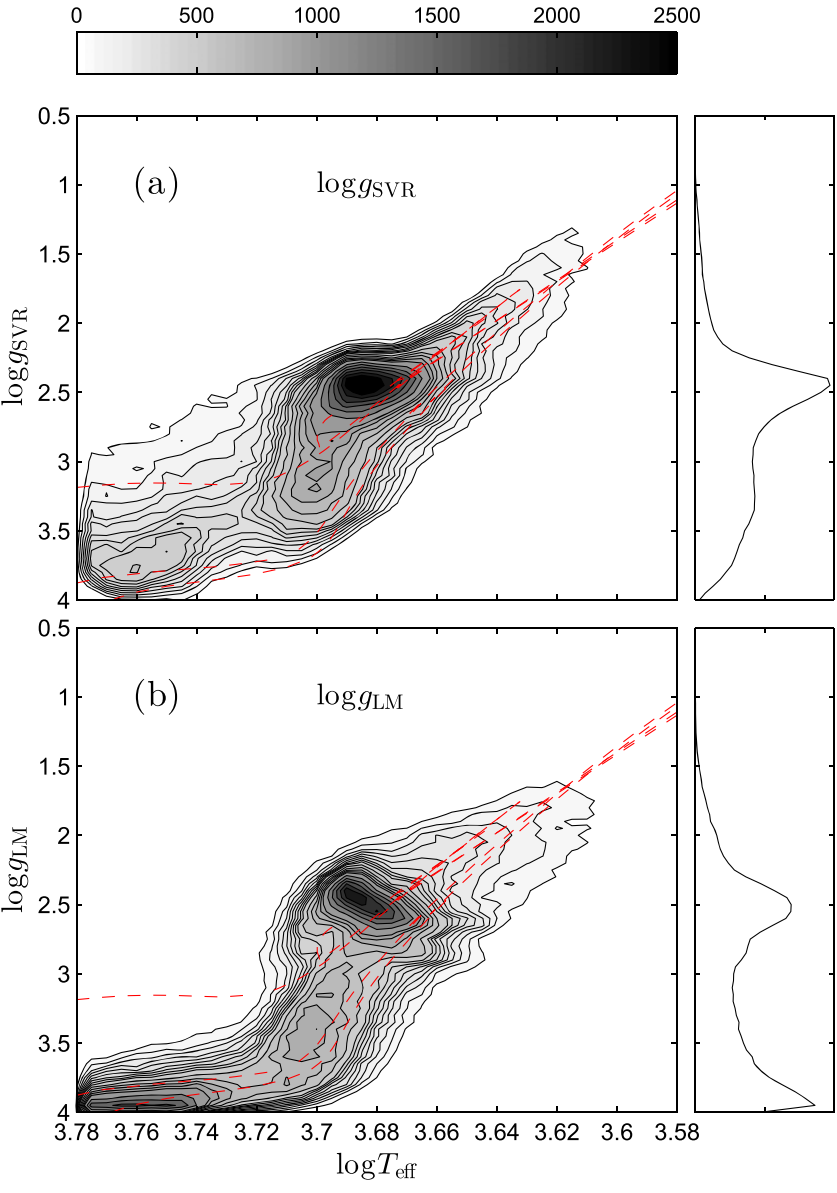

Figure 11. (a) Distribution of the LAMOST metal-rich giant stars in $\log T_{\text {eff }}$ vs. $\log g$ plane. The $T_{\text {eff }}$ is from the LAMOST pipeline, while the $\log g$ is estimated from the seismic-based SVR. The contours indicate the density. (b) Same distribution for the same data set as (a), but with the LAMOST $\log g$. The red dashed lines show the isochrones with $[\mathrm{Fe} / \mathrm{H}]=-0.2$ dex at the age of 1,5 , and $10 \mathrm{Gyr}$, from top to bottom. The distributions of the two estimated $\log g$ are shown in the right panels as the black lines.

$\log g$. We refer the reader to Chaplin \& Miglio (2013) for a detailed review.

According to Equation (3), the accuracy of the surface gravity depends on a few factors: the frequency of maximum power $\nu_{\max }$, the effective temperature $T_{\text {eff }}$, and the corresponding solar parameters. Gai et al. (2011) investigated the grid-based method to derive stellar properties, including $\log g$, from seismic parameters combined with effective temperature and metallicity. They found that the uncertainty (in terms of FWHM of the distribution of the fractional deviation of the true value) is smaller for stars with large $\log g$ than for those with small $\log g$. In all cases, the uncertainty is within $5 \%$ of the true $\log g$. Huber et al. (2012) combined the asteroseismology, interferometry, and parallax of the stars and found that the uncertainties of the seismic scaling relations are in agreement with other measurements, and the uncertainties do not depend on the evolution stage of the stars. However, Hekker et al. (2013) inferred that the seismic parameter determinations give small systematic bias in $\log g$ for both red clump and RGB stars, although the extent of the systematics is within 0.01 dex. More investigations about the various evolution phases of stars are given in Section 4.2.

All these systematic biases do affect the SVR estimates for the LAMOST data, since the SVR treats the seismic $\log g$ as the standard. Therefore, the users who intend to apply the $\log g_{\text {SVR }}$ in any study of the structure and evolution of the Milky Way should very carefully validate whether these systematic biases affect their results.

It is also worthwhile to note that the limit of the techniques and observations in asteroseismology may bias the sampling of the giant stars at different $\log g$. For the giant stars with larger $\log g$, because their oscillation amplitude is very small (Huber et al. 2011) and thus hard to detect, many of these kinds of stars in the Kepler field lack asteroseismic $\log g$. On the other hand, the giant stars with smaller $\log g$ are also undersampled because their oscillation frequency is too low and cannot be reliably measured during the $4 \mathrm{yr}$ observations with Kepler. Therefore, the training data set lacks data at both ends of the distribution of the $\log g_{\text {ast }}$. This may lead to some systematics in the SVR method because of the imbalance of the training data set. Fortunately, it seems that the training data set contains sufficient stars with either very small or very large $\log g_{\text {ast }}$ and the systematic bias shown in panel (c) of Figure 10 is acceptable, compared to the uncertainty of $\sim 0.1 \mathrm{dex}$.

\subsection{Effect of Evolution Phases}

Pinsonneault et al. (2014) found that the spectroscopic and seismic $\log g$ are systematically different for the giant stars in different evolution stages, e.g., the primary red clump stars, 


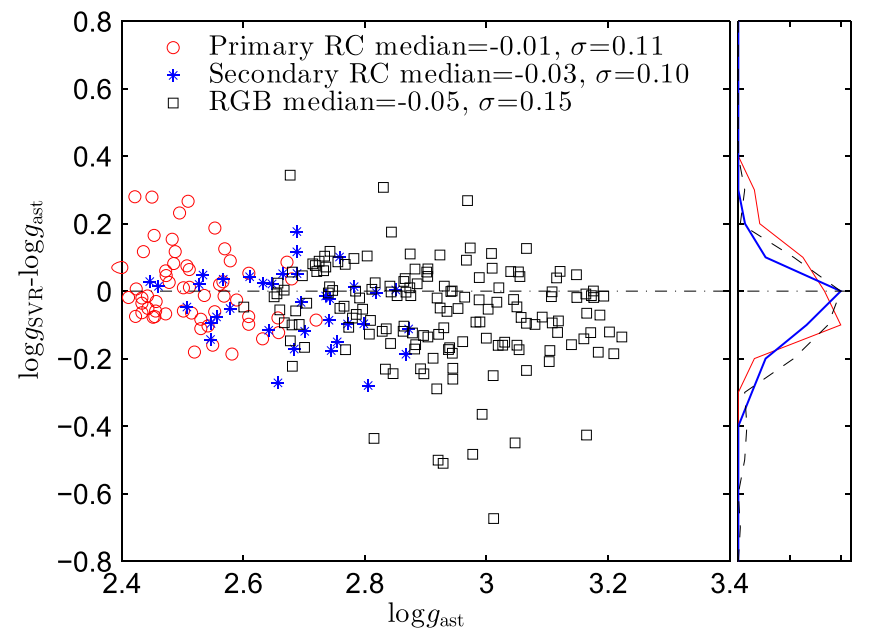

Figure 12. Residual $\log g$ as a function of the seismic $\log g$ for the primary red clump stars (red circles), secondary red clump stars (asterisks), and RGB stars (black circles). The right panel shows the distribution of the three types of stars with the same colors as the main plot.

secondary red clump stars, ${ }^{9}$ and RGB stars. Although the training data set of the SVR model uses seismic $\log g$, the input data are based on the spectra. Therefore, it may also have a similar systematic bias to that shown in Figure 3 of Pinsonneault et al. (2014). We then tag the primary and secondary red clump stars and RGB stars in the test data set by cross-identifying it with the data from Stello et al. (2013), in which the evolution phases are distinguished by the period spacings of the dipole mode. Figure 12 shows that $\log g$ SVR is about 0.05 dex lower than $\log g_{\text {ast }}$ for the RGB stars. Although almost no overall systematics is found for primary red clump stars, a few of them are overestimated by about 0.2 dex (see the left side of Figure 12). For the secondary red clump stars, the median residual slightly shifts to -0.03 dex. This can explain why in panel (c) of Figure 10 the SVR $\log g$ is systematically underestimated for stars with $\log g_{\text {ast }}>3$ dex, which are mostly RGB stars, and then slightly overestimated for $\log g_{\text {ast }}<2.25$ dex. Moreover, the dispersion of the residual between $\log g_{\text {SVR }}$ and $\log g_{\text {ast }}$ is larger in RGB stars than in red clump stars. It seems that the effect of evolution phases in LAMOST is different from that in the APOGEE data (Pinsonneault et al. 2014). In future works, more investigations will be necessary to address the reason why the different evolution stages show different biases in $\log g$ estimates and to cross-calibrate the parameters between LAMOST and APOGEE data.

\subsection{Metallicity}

Currently, the seismic $\log g$ is limited to the metal-rich stars. This is because the empirical scaling relation used in the asteroseismology measurement is based on the solar-type stars. Epstein et al. (2014a) found that the seismic mass estimation for the halo and thick-disk stars is significantly higher than the expectations. Although the stellar radius estimation is more

\footnotetext{
9 The primary red clump stars are the He core burning giant stars with electronic-degenerate cores, while the secondary clump stars are massive enough to have non-degenerate He-cores. In general, the primary clump stars are low-mass and hence older. However, the secondary clump stars are younger, and the present stellar model predicts that they are around $1 \mathrm{Gyr}$ old (Girardi 1999).
}

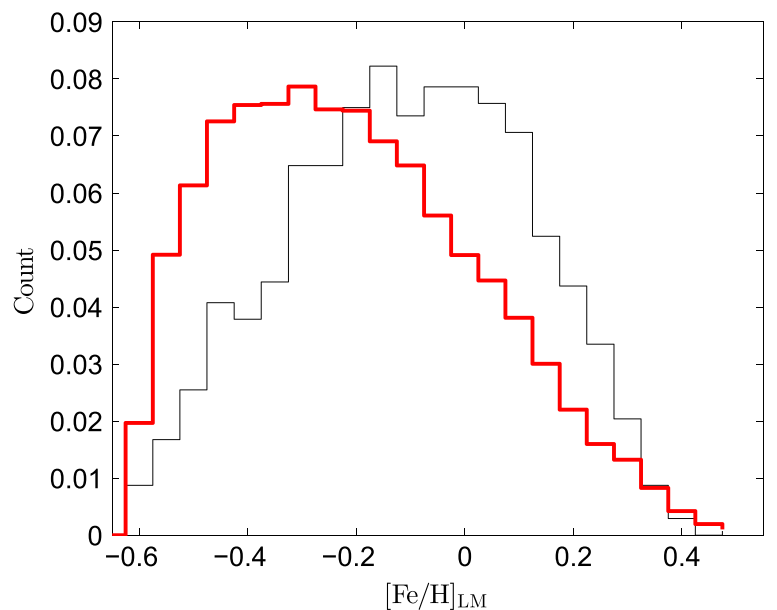

Figure 13. Metallicity distribution of the training data set (black line) and the LAMOST DR2 data (red line). The $y$-axis is the count of stars normalized by 1 .

precise than the stellar mass (Gai et al. 2011), it may also be affected by the same systematic bias. Therefore, we only apply the seismic-trained SVR model to the metal-rich giant stars $([\mathrm{Fe} / \mathrm{H}]>-0.6 \mathrm{dex})$ to avoid the probably systematic shift for the metal-poor stars.

It is also worthwhile to compare the metallicity distribution functions (MDFs) of the training data set and the selected LAMOST data to ensure that the training data set covers the entire range of the metallicity of the LAMOST data. Figure 13 shows the two MDFs (the gray and red lines stand for the MDF of the training data set and the LAMOST data, respectively). Because both samples are cut at -0.6 dex, the two MDFs are truncated at this value. Although the peaks of the two distributions are different by about 0.2 dex, they approximately cover the same range of metallicity, except a very small fraction of data at about 0.4 dex. Therefore, the scaling relation of the seismology used in the surface gravity estimation for the training data set is also suitable for the selected LAMOST data.

Moreover, panel (b) of Figure 10 shows that $\log g_{\text {SVR }}$ does not strongly correlate with metallicity. Therefore, the slight difference of the MDFs shown in Figure 13 would not lead to significant systematics in the derived $\log g$ for the LAMOST data.

\subsection{Comparison with Other Works}

Mészáros et al. (2013) and Holtzman et al. (2015) calibrated the surface gravity of the APOGEE (Ahn et al. 2014) data to seismic values with simple functions. Compared to the technique employed in this work, their methods are direct and simple. However, the measurement errors in their methods are contributed twice from two different processes: one is from the estimation of the initial $\log g$ based on a synthetic library, and the other is from the calibration after $\log g$ estimation. Therefore, the uncertainty of the calibrated $\log g$ may be intrinsically larger. In our method, the seismic $\log g$ is directly used as the standard values in the training process, and therefore the measurement error is only produced once during the determination of the $\log g$. The derived $\log g$ from the SVR model is naturally calibrated with the seismic $\log g$. In other words, the SVR model merges the two steps of determination and calibration of $\log g$ into one single step and hence avoids additional error contribution. 


\subsection{Degeneracies between $\log \mathrm{g}, \mathrm{T}_{\mathrm{eff}}$, and $[\mathrm{Fe} / \mathrm{H}]$}

Figures 4-6 show that the stellar parameters, $\log g, T_{\text {eff }}$, and $[\mathrm{Fe} / \mathrm{H}]$, are not precisely orthogonal in the spectral lines. In some of the lines, the stellar parameters even change toward similar directions, producing some extents of degeneracies. It is quite difficult to completely break the degeneracies in a stellar parameterization technique, particularly in an algorithm like SVR, which can only determine one parameter at a time. Indeed, Figure 10 does show that the residuals of $\log g_{\mathrm{SVR}}$ bias from zero by at most $\sim 0.05 \mathrm{dex}$ in $T_{\text {eff }}$ and $[\mathrm{Fe} / \mathrm{H}]$. However, considering that the $\log g_{\mathrm{SVR}}$ estimated in this work will be mainly used in the large sample statistics with tens or even hundreds of thousands of stars in the Galaxy, these weak biases may not significantly affect the results.

It is also noted that the SVR $\log g$ may also change the estimation of $T_{\text {eff }}$ and $[\mathrm{Fe} / \mathrm{H}]$. Figures 4-6 show that some spectral lines are sensitive to all three parameters. This means that when we estimate $T_{\text {eff }}$ and $[\mathrm{Fe} / \mathrm{H}]$ with the adopted SVR $\log g$ values, the derived $T_{\text {eff }}$ and $[\mathrm{Fe} / \mathrm{H}]$ may be quite different from the current values from the LAMOST pipeline. The difference can be quantified by reestimating the $T_{\text {eff }}$ and $[\mathrm{Fe} / \mathrm{H}]$ in the LAMOST pipeline with the fixed SVR $\log g$ value. This, however, is beyond the scope of this paper and should be investigated in future works.

\subsection{Benefits from Accurate $\log \mathrm{g}$}

We can significantly improve the accuracy of the distance estimation from accurate $\log g$ estimates. Since the stellar luminosity follows $L \propto R^{2}$ and surface gravity follows $g \propto R^{-2}$, we have $L \propto g^{-1}$. Consequently, the absolute magnitude $M$ is proportional to $\log L \sim 2.5 \log g$. This means that when the uncertainty of $\log g$ is $0.1 \mathrm{dex}$, the uncertainty of the absolute magnitude turns out to be $0.25 \mathrm{mag}$, which is equivalent with about $12 \%$ in distance. ${ }^{10}$ This is a factor of $\sim 2$ better than the accuracy of distance derived from the non-seismic-based spectroscopic $\log g$ (Carlin et al. 2015).

\subsection{The Dwarf Stars}

The known Kepler seismic $\log g$ are mostly for the giant stars (Huber et al. 2014). Hence, we lack dwarf star samples as the training data set. As a consequence, we only apply the method to giant stars and do not expand it to dwarf stars. The LAMOST $\log g$ is sufficiently accurate for the separation of the giant and dwarf stars. Therefore, we use it to select the giant stars first and let the seismic-trained SVR model predict more accurate $\log g$ for the selected giant stars. In the future, the coming PLATO mission may provide another tens of thousands of asteroseismic measurements over the entire HertzsprungRussell diagram (Rauer et al. 2014). This will help to enrich the dwarf training data set and expand this work to the dwarf stars.

\section{CONCLUSIONS}

Although we use the LAMOST spectra as the training data set, it does not limit the application of the SVR model only to the LAMOST data. In general, given other spectroscopic survey data without seismic measurement, we can first calibrate

\footnotetext{
${ }^{10}$ Note that the accuracy of the distance does not take into account the uncertainties contributed by photometries, e.g., the coarse dereddening, less accurate magnitude, etc.
}

the equivalent widths of the line indices to align with LAMOST and then apply the model to the new data. Indeed, we test it using a set of MMT/Hectospec-observed giant stars, in which more than 100 common objects with LAMOST are found. The accuracy of the $\log g$ estimates for these samples is roughly at the same level as in this work (more details will be given in C. Liu et al. 2015, in preparation).

In summary, although not all LAMOST data have asteroseismic observations, we can use a small subset with the Kepler seismic $\log g$ as the training data set to estimate $\log g$ for other LAMOST data with an SVR model. The approach can reach to an accuracy as high as $0.1 \mathrm{dex}$ when the $\mathrm{S} / \mathrm{N}$ of the spectra is higher than 20 . This improves the current $\log g$ estimated from the LAMOST pipeline by at least a factor of 2. This significant improvement will be very useful in the following studies: (1) it allows us to better estimate the distance of the giant stars with accuracy of about $12 \%$, and (2) it enables us to separate the primary and secondary red clump stars from $\log g$, providing good samples to trace the stellar populations with different ages.

We thank the anonymous referee for useful comments. We also thank René Andrae, Coryn Bailer-Jones, and Shude Mao for the helpful comments and discussions. This work is supported by the Strategic Priority Research Program "The Emergence of Cosmological Structures" of the Chinese Academy of Sciences, grant No. XDB09000000, and the National Key Basic Research Program of China 2014CB845700. C.L. acknowledges the National Science Foundation of China (NSFC) under grants 11373032, 11333003, and U1231119. M.F. acknowledges the NSFC under grant 11203081. Y.W. acknowledges the NSFC under grant 11403056. Guoshoujing Telescope (the Large Sky Area Multi-object Fiber Spectroscopic Telescope: LAMOST) is a National Major Scientific Project built by the Chinese Academy of Sciences. Funding for the project has been provided by the National Development and Reform Commission. LAMOST is operated and managed by the National Astronomical Observatories, Chinese Academy of Sciences.

Facilities: LAMOST.

\section{REFERENCES}

Ahn, C. P., Alexandroff, R., Allende Prieto, C., et al. 2014, ApJS, 211, 17 Borucki, W. J., Koch, D., Basri, G., et al. 2010, Sci, 327, 977

Brown, T. M., \& Gilliland, R. L. 1994, ARA\&A, 32, 37

Burges, C. J. C. 1998, Data Mining Knowledge Discovery, 2, 121

Carlin, J. L., Liu, C., Newberg, H. J., et al. 2015, AJ, in press (arXiv:1505.05521)

Cassisi, S., \& Salaris, M. 1997, MNRAS, 285, 593

Chang, C.-C., \& Lin, C.-J. 2011, ACM Transactions on Intelligent Systems and Technology, 2, 27

Chaplin, W. J., Bedding, T. R., Bonanno, A., et al. 2011, ApJL, 732, L5

Chaplin, W. J., \& Miglio, A. 2013, ARA\&A, 51, 353

Cortes, C., \& Vapnik, V. 1995, Mach. Learn., 20, 273

Cui, X.-Q., Zhao, Y.-H., Chu, Y.-Q., et al. 2012, RAA, 12, 1197

Creevey, O. L., Thévenin, F., Basu, S., et al. 2013, MNRAS, 431, 2419

De Cat, P., Fu, J.-N., Ren, A. B., et al. 2014, ApJS, submitted

Deng, L.-C., Newberg, H. J., Liu, C., et al. 2012, RAA, 12, 735

Deng, N., Tian, Y., \& Zhang, C. 2012, Support Vector Machines: Optimization Based Theory, Algorithms, and Extensions (London/Boca Raton, FL: Chapman and Hall/CRC)

Drucker, H., Burges, J. C. C., Kaufman, L., Smola, A., \& Vapnik, V. 1996, Advances in Neural Information Processing Systems, 9, 155

Epstein, C. R. 2014b, arXiv:1409.2277

Epstein, C. R., Elsworth, Y. P., Johnson, J. A., et al. 2014a, ApJL, 785, L28 Gai, N., Basu, S., Chaplin, W. J., \& Elsworth, Y. 2011, ApJ, 730, 63 
Girardi, L. 1999, MNRAS, 308, 818

Hekker, S., Elsworth, Y., Basu, S., et al. 2013, MNRAS, 434, 1668

Holmberg, J., Nordström, B., \& Andersen, J. 2007, A\&A, 475, 519

Holtzman, J. A., Shetrone, M., Johnson, J. A., et al. 2015, AJ, submitted (arXiv: 1501.04110$)$

Huber, D., Bedding, T. R., Stello, D., et al. 2011, ApJ, 743, 143

Huber, D., Ireland, M. J., Bedding, T. R., et al. 2012, ApJ, 760, 32

Huber, D., Silva Aguirre, V., Matthews, J. M., et al. 2014, ApJS, 211, 2

Lee, Y. S., Beers, T. C., Sivarani, T., et al. 2008, AJ, 136, 2022

Lenz, D. D., Newberg, H. J., Rosner, R., Richards, G. T., \& Stoughton, C. 1998, ApJS, 119, 121

Liu, C., Bailer-Jones, C. A. L., Sordo, R., et al. 2012, MNRAS, 426, 2463

Liu, C., Deng, L.-C., Carlin, J. L., et al. 2014, ApJ, 790, 110

Majewski, S. R., Ostheimer, J. C., Kunkel, W. E., \& Patterson, R. J. 2000, AJ, 120,2550

Majewski, S. R., Wilson, J. C., Hearty, F., Schiavon, R. R., \& Skrutskie, M. F. 2010, in IAU Symp. 265, Chemical Abundances in the Universe: Connecting First Stars to Planets, ed. K. Cunha, M. Spite, \& B. Barbuy (New York, NY: AIP), 480481

Marigo, P., Girardi, L., Bressan, A., et al. 2008, A\&A, 482, 883
Mészáros, S., Holtzman, J., García Pérez, A. E., et al. 2013, AJ, 146, 133

Morel, T., \& Miglio, A. 2012, MNRAS, 419, 34

Morrison, H. L., Norris, J., Mateo, M., et al. 2003, AJ, 125, 2502

Pinsonneault, M. H., An, D., Molenda-Żakowicz, J., et al. 2012, ApJS, 199, 30

Pinsonneault, M. H., Elsworth, Y., Epstein, C., et al. 2014, ApJS, 215, 19

Rauer, H., Catala, C., Aerts, C., et al. 2014, ExA, 38, 249

Re Fiorentin, P., Bailer-Jones, C. A. L., Lee, Y. S., et al. 2006, A\&A, 467, 1373

Smola, A. J., \& Schölkopf, B. 2004, Statistics and Computing, 14, 199

Stello, D., Huber, D., Bedding, T. R., et al. 2013, ApJL, 765, L41

Wilhelm, R., Beers, T. C., \& Gray, R. O. 1999, AJ, 117, 2308

Worthey, G., Faber, S. M., Jesus Gonzalez, J., \& Burstein, D. 1994, ApJS, 94, 687

Worthey, G., \& Ottaviani, D. L. 1997, ApJS, 111, 377

Wu, Y., Luo, A., Du, B., Zhao, Y., \& Yuan, H. 2014, arXiv:1407.1980

Wu, Y., Luo, A.-L., Li, H.-N., et al. 2011a, RAA, 11, 924

Wu, Y., Singh, H. P., Prugniel, P., Gupta, R., \& Koleva, M. 2011b, A\&A, 525, 7

Xue, X.-X., Ma, Z., Rix, H.-W., et al. 2014, ApJ, 784, 170

Yanny, B., Newberg, H. J., Kent, S., et al. 2000, ApJ, 540, 825

Zhao, G., Zhao, Y.-H., Chu, Y.-Q., et al. 2012, RAA, 12, 723 